\title{
Robustness in angiogenesis: Notch and BMP shaping waves
}

\author{
Karen Beets ${ }^{1,2}$, Danny Huylebroeck ${ }^{3}$, Iván M. Moya ${ }^{1,2}$, Lieve Umans ${ }^{1,2,3}$, and \\ An Zwijsen ${ }^{1,2}$
}

\author{
${ }^{1}$ Laboratory of Developmental Signaling, VIB Center for the Biology of Disease, VIB, 3000 Leuven, Belgium \\ ${ }^{2}$ Center for Human Genetics, KU Leuven, 3000 Leuven, Belgium \\ ${ }^{3}$ Laboratory of Molecular Biology (Celgen), Department of Development and Regeneration, KU Leuven, 3000 Leuven, Belgium
}

\begin{abstract}
Vascular patterning involves sprouting of blood vessels, which is governed by orchestrated communication between cells in the surrounding tissue and endothelial cells (ECs) lining the blood vessels. Single ECs are selected for sprouting by hypoxia-induced stimuli and become the 'tip' or leader cell that guides new sprouts. The 'stalk' or trailing ECs proliferate for tube extension and lumenize the nascent vessel. Stalk and tip cells can dynamically switch their identities during this process in a Notch-dependent manner. Here, we review recent studies showing that bone morphogenetic protein (BMP) signaling coregulates Notch target genes in ECs. In particular, we focus on how Delta-like ligand 4 (DLL4)-Notch and BMP effector interplay may drive nonsynchronized oscillatory gene expression in ECs essential for setting sharp tip-stalk cell boundaries while sustaining a dynamic pool of nonsprouting ECs. Deeper knowledge about the coregulation of vessel plasticity in different vascular beds may result in refinement of antiangiogenesis and vessel normalization therapies.
\end{abstract}

\section{Development of a branched vascular tree}

The proper formation of blood vessels is critical for developmental growth as well as for tissue growth and physiology beyond birth. The blood vessel circulatory system comprises arteries, capillaries, and veins, and it fuels nearly every tissue with oxygen and nutrients, clears tissues of metabolic waste, and transports liquids and cells. Failure to establish a hierarchical branched vessel network leads to embryonic lethality, whereas deregulation of vessel patterning after birth contributes to the development of cancer and to chronic and inflammatory diseases.

Blood vessel networks form through vasculogenesis, a de novo assembly process, as well as through angiogenesis, which is the coordinated expansion of a pre-existing vascular network [1] (see Glossary). New blood vessels form by angiogenesis through the splitting of pre-existing vessels (intussusceptions) or through sprouting [1]. We focus here on the morphologically and molecularly best-understood process, sprouting angiogenesis. This highly orchestrated multistep process initiates when ECs in vessels become induced to form new sprouts that directionally migrate

Corresponding author: Zwijsen, A. (an.zwijsen@cme-vib.kuleuven.be) Keywords: angiogenesis; endothelial cell competence; hypersprouting; lateral inhibition; oscillatory gene expression; plasticity. towards a hypoxic area; the process further involves branch extension, acquisition of a lumen, new connection formation (anastomosis), and vessel maturation. Vessel maturation encompasses recruitment of mural cells, strengthening of cell-cell junctions, and deposition of a basement membrane. Excessive vessels can also be pruned (vessel regression). Together, these processes lead to a functional and hierarchical network of vessels that remains adjustable, expanding or regressing depending on the oxygen needs (reviewed in [1]). Efficient and robust sprouting depends on induction of sufficient but not excessive numbers of ECs to become tip cells. An unbalanced ratio of tip:stalk cells results in excessive sprouting (hypersprouting) or paucity of sprouting.

Two EC phenotypes play a prominent role during sprouting angiogenesis: the tip cell and the stalk cell. The tip cell is the leader selected to spearhead the emerging sprout. This cell extends filopodia, is migratory, polarized, and pulls the stalk cells into the emerging branch.

\section{Glossary}

Angiogenesis: the formation of new blood vessels from pre-existing vessels by vessel sprouting (sprouting angiogenesis) or splitting (intussusception). Endothelial cells (ECs): cells that line the interior surface of blood and lymphatic vessels.

Lateral inhibition: an event in which a cell adopts a particular phenotype and prevents its immediate neighbor from acquiring the same phenotype through Notch-mediated signaling.

Morphogen: a signaling factor that elicits different cellular responses in responding cells depending on its concentration. During early development, morphogen gradients generate different cell types in a distinct spatial order and pattern in embryos and/or tissues.

Mural cells: cells covering the endothelial cell tube. Mural cells stabilize nascent vessels, provide support, and guide remodeling. Pericytes are the mural cells in the microvasculature, whereas vascular smooth muscle cells cover larger vessels.

Nonsynchronized oscillatory expression: occurs when cells in a tissue exhibit oscillatory gene expression (e.g., HES1 or $I D$ ), but the individual cells are in different phases of their cycle. These nonsynchronous oscillations permit differential cellular responses and behavior in a population of cells that is exposed to the same stimulus.

Phalanx cell: quiescent EC.

Somite: segmental axial structures in the embryo that give rise to the vertebral column, ribs, skeletal muscles, and dermis.

Stalk cell: the ECs that trail behind the tip cell. These follower cells proliferate, extend the sprout, and form the lumen in nascent vessels.

Tip cell: this leader EC extends filopodia and is migratory and polarized. The tip cell pulls the stalk cells into the emerging sprout and guides the sprout. Ultradian oscillatory expression: gene expression occurring in periods or cycles that are repeated frequently (i.e., every $2 \mathrm{~h}$ ) throughout a 24-h period. Vasculogenesis: de novo formation of blood vessels from the local differentiation of ECs. 
The stalk or follower cells trail the tip cell; they proliferate, extend the sprout, and form the lumen in nascent vessels. A third cell type, the phalanx cell, is a quiescent EC in a stable vessel. Each of these cell types has specific features and is enriched in a specific gene transcript repertoire [2,3]. Vascular endothelial growth factor (VEGF) is a potent proangiogenic stimulus that induces the selection of single tip cells by enrichment of membrane-bound DLL4, a ligand for the receptor Notch, through activation of some of its receptors, VEGFR2 and especially VEGFR3 [4,5]. This results in activation of Notch-1 in adjacent ECs (Box 1), thereby triggering the induction of the stalk-cell phenotype via lateral inhibition in these cells (reviewed in [3]).

The plasticity of ECs is extraordinary; individual ECs have the intrinsic capacity to become tip, stalk, and phallanx cells in a context-dependent manner. Furthermore, tip and stalk cells have been demonstrated to shuffle and actively take over the position and identity of the other, which requires the swift induction and repression of transcripts specific for either phenotype. The competition in the rush for the tip cell position depends on relative DLL4Notch signaling levels that control expression levels of VEGFR1, VEGFR2, and VEGFR3 [5,6]. It appears that ECs dynamically re-evaluate their neighbors by interpreting numerous extracellular pro- and anti-angiogenesis and vascular guidance cues provided by the surrounding tissue, as well as signals that emanate in the ECs, and then use this information to respond to their environment. VEGF not only is a crucial inducer of the tip cell phenotype and migration, but also regulates stalk cell proliferation, whereas DLL4-Notch signaling and its crosstalk to VEGF

\section{Box 1. The Notch signaling cascade in endothelium}

Notch-mediated signaling is a highly conserved signaling system that controls many differentiation processes during development (Box 2) [34]. The principal roles of Notch signaling in endothelium are arterial specification, lateral inhibition of new sprout formation during angiogenesis, and control of blood vessel quiescence. Studies from mouse retina models and in zebrafish embryos, tumor models, and cell culture 3D sprouting assays have established that DLL4-Notch signaling serves as a negative regulator of VEGFinduced angiogenesis, and that inhibition of the Notch pathway leads to excessive sprouting (hypersprouting) of blood vessels [79]. Endothelium expresses mainly Notch 1 and Notch 4 receptors, which become activated in the signal-receiving EC by membraneembedded DLL4 or JAG1 ligands presented by adjacent signal sending cells. This leads ultimately to cleavage of the Notch receptor at the juxtamembrane domain by $\gamma$-secretase to release the NICD, which translocates into the nucleus where it is recruited to target genes via interaction with the DNA-binding protein CSL/RBPJ/ CBF1 (see Figures 1 and 2a in main text) [34]. This complex recruits additional transcriptional activators and induces target genes, such as Hes1 and Hey, forming the Notch-CSL-Hes axis (Box 2). HES1 and HEY/HERP belong to families of unstable bHLH transcriptional repressors that function in maintaining progenitor cells (including adult stem cells) and regulate binary cell fate decisions [86]. HES proteins are class $C$ transcriptional repressors, binding not only to class $C$ sites (CACGNG) and N-box sequences (CACNAG), but also class $B$ sites. The HERP family of bHLH proteins binds both class $B$ and $C$ sites $[87,88]$. HES proteins have been documented to repress transcriptionally several genes known to control EC phenotypes and tip-stalk cell selection (e.g., VEGFR2 and -3, DLL1, DLL4, and JAG1) $[54,66,89]$. Heterozygous DI/4 gene deletion in mice or inhibition of Notch1 cleavage with $\gamma$-secretase inhibitors results in excessive sprouting of the developing vascular network [65]. balances the tip:stalk cell ratio. Several excellent and comprehensive reviews are dedicated to more in-depth discussion on the tight link between VEGF and Notch signaling in vascular development [7-9]. Here, we discuss recent studies that show that another signaling pathway, elicited by BMPs, collaborates with DLL4-Notch signaling to balance the tip:stalk cell ratio, and that tip and stalk cell competence critically depends on nonsynchronized oscillatory levels of BMP-SMAD and Notch signaling effectors.

\section{An introduction to BMP signaling}

BMPs are secreted ligands of the transforming growth factor $\beta$ (TGF- $\beta$ ) family, which also includes TGF- $\beta$, BMPs, growth, and differentiation factors (GDFs), activins, and nodal. The BMPs can be subdivided based on their structural properties and documented ligand-receptor interactions into four groups: (i) the BMP2 and -4 subgroup; (ii) the growth and differentiation factor (GDF) $5,-6$, and, -7 group; (iii) the BMP5, -6, -7, and -8 group; and (iv) the BMP9 and 10 group (reviewed in [10]). BMPs were first identified as potent inducers of ectopic bone formation when implanted subcutaneously on appropriate carriers [11,12], and recombinant BMPs are being used in the clinic to treat nonunion bone fractures and kidney disorders. However, BMPs turned out to be far more versatile than their name suggests. They regulate a multitude of functions at the cellular level, such as pluripotency, cell-type specification and differentiation, proliferation and migration, and apoptosis (reviewed in [10,13]). Altogether, multitasking BMPs are critical for the embryonic development of many organs and adult tissue homeostasis, and hence, it has been suggested that BMPs be renamed 'body morphogenetic proteins' [13].

BMPs signal via binding to heteromeric combinations of type I and II transmembrane serine/threonine kinase type receptors (Figure 1). Most receptors display a broad BMP specificity, which results in a great combinatorial complexity of ligand-receptor partners [14]. An interesting exception is the activin receptor-like kinase 1 (ALK1), which is a type I receptor largely confined to endothelium that exhibits striking selectivity for BMP9 and BMP10. Likewise, BMP9 and -10 appear to signal almost exclusively via this type I receptor in ECs [15-17]. BMP-receptor binding triggers phosphorylation of the receptor-regulated SMADs (R-SMADs), SMAD1, -5 and -8, in their C-terminal segment, initiating the canonical or SMAD-mediated pathway. In many cell culture systems and in the mouse vasculature, SMAD1 and -5 function redundantly [18$20]$, although they are each essential during early mouse development [21]. SMAD8 is, unlike SMAD1 or -5, dispensable during development [21]. Interestingly, recent studies showed a nonredundant function of SMAD8 in miRNA processing required for BMP-induced growth suppression [22], and association of SMAD8 mutations with pulmonary arterial hypertension $[23,24]$. Other SMADs that participate in BMP signaling include the common-mediator SMAD (co-SMAD) SMAD4 and the inhibitory SMADs (ISMADs), SMAD6 and SMAD7 [25,26] (Figure 1).

Once activated, phosphorylated (P)SMAD1, -5 , and -8 form hetero-oligomeric SMAD complexes with SMAD4 that accumulate in the nucleus, where they positively or negatively regulate transcription of target genes [27] 


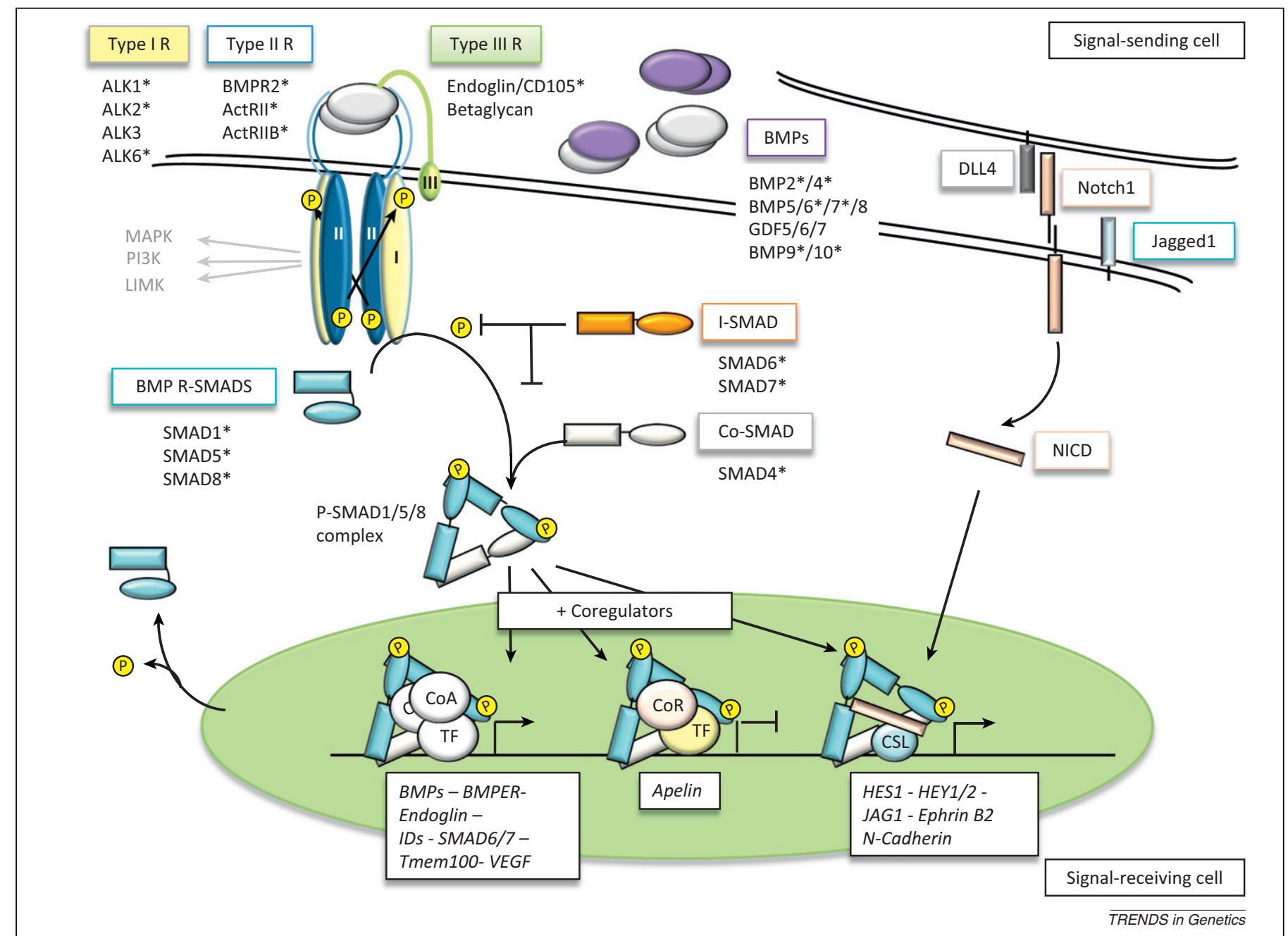

Figure 1. BMP-SMAD and Notch signaling pathways converge in endothelial cells (ECs). Schematic layout of BMP-SMAD and DLL4-Notch mediated signaling pathways and their integration. The BMP-SMAD signaling pathway is represented in greater detail; the asterisks $(*)$ indicate pathway components shown to be functional in ECs. BMP

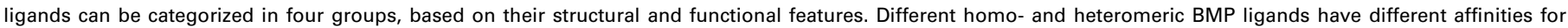
different receptors complexes. Major BMPs in endothelium are BMP2, $-4,-6,-7,-9$, and -10 . BMP ligands signal through their interaction with a tetrameric receptor complex comprising two type I and two type II serine/threonine kinase receptors. BMPR2, ActRII, and ActRIIB are type II receptors that bind BMP ligands, whereas ALK1, -2 , -3 , and -6 are BMP type I receptors [90,91]. Unlike most BMPs that have pleiotropic functions in different cell types and interact with different receptor complexes, the BMP9 and -10 ligands interact almost exclusively with EC-specific ALK1-encompassing receptor complexes. BMP9 and -10 are involved in maintenance of quiescence, whereas BMP2, -4 , 6 , and -7 are implicated in migration and angiogenesis [36]. Type III receptors are auxiliary coreceptors that can modify ligand-receptor affinities. Endoglin/CD105 can modulate BMP-ALK1 effects in ECs, but activin and TGF $\beta 1$ and -3 have also been shown to bind endoglin in combination with other type I receptors. ALK1, BMPR2, and endoglin are critical BMP receptors in ECs. Type II receptors are constitutively active kinases that phosphorylate the GS box (SGSGSG-motif) of type I receptors upon ligand binding. This activates the kinase activity of the latter and exposes a binding site for its main substrates, the R-SMADs. BMP-receptor binding triggers C-terminal phosphorylation of R-SMADs (canonical signaling) or nonSMAD-mediated signaling (reviewed in [92-94]); the latter mode of signaling is not further detailed here because their role in vascular development is largely unclear and there is no obvious link between nonSMAD and Notch signaling. SMAD1, -5 , and -8 mediate BMP signaling, whereas SMAD2 and -3 (not pictured) are R-SMADs specific for TGF $\beta$-nodal-activin signaling. Phosphorylated R-SMADs form complexes with SMAD4 and translocate into the nucleus, where they can interact with different co-activators, repressors, and eventually transcription factors. Examples of BMP-SMAD target genes in ECs are listed. Several genes, including well-known Notch target genes, have been demonstrated to be directly induced by BMP-SMAD ECs (reviewed in [27]). Indirect regulation of transcription occurs through interaction with DNA-binding transcription factors and histone modifying factors and/or complexes [27]. Notch receptors become activated in the signal receiving EC by membrane-embedded DLL presented by adjacent signal sending cells. This leads to proteolytic cleavage of the Notch receptor and release of the NICD, which translocates to the nucleus, where it is recruited to target genes via interaction with the DNA-binding protein CSL/RBPJK/CBF1. This complex recruits additional transcriptional activators and induces target genes, such as $H E S$ and $H E Y$ bHLH transcriptional repressors, forming the Notch-CSL-HES axis. There is compelling evidence that Notch and BMP-SMAD cascades cooperatively induce in ECs HES1, HEY1, HEY2, JAG1, and the gene encoding N-cadherin (CDH1) [20,38,49-51,56], whereas BMPSMADs induce ID, SMAD6, and -7 in an NICD-independent fashion. Expression of the gene encoding ephrin-B2 (EFNB2) has been shown to be dependent on ID-mediated BMP9-ALK1 signaling [95] and is also induced by VEGF-DLL4-Notch; therefore, it is classified here with the Notch and BMP-SMAD-dependent target genes. Remarkable few genes have been reported in ECs to be repressed by BMP-SMADs; it remains to be resolved how BMP-SMAD-mediated repression of the gene encoding apelin (APLN) occurs [38,96]. BMP transcription is under direct autoregulation (feedforward regulation), and BMP signaling also induces positive and negative feedback mechanisms via the induction of the inhibitory SMADs (SMAD6, and -7) and extracellular antagonists, such as BMPER, respectively. SMAD6 and -7 inhibit the BMP-SMAD and TGF-SMAD axes, respectively. Recently, SMAD8 has been demonstrated to also control miRNA processing [22], but this novel activity is not represented here. Abbreviations: ActR, activin receptor; ALK1, activin receptor-like kinase 1; (b)HLH, (basic) helix-loop-helix; BMP, bone morphogenetic protein; BMPR2, BMP receptor 2; BMPER, BMP binding

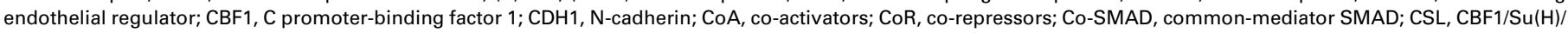
Lag-1 family transcription factor complex; DLL4, delta-like 4; EC, endothelial cell; HES1, hairy and enhancer of split 1; GDF, growth/differentiation factor; HEY, hairy/ enhancer-of-split related with YRPW motif; ID, inhibitors of differentiation or inhibitor of DNA-binding proteins; I-Smad, inhibitory SMAD; JAG1, jagged 1; LIMK, LIM domain kinase; MAPK, mitogen-activated kinase; NICD, Notch intracellular domain; PI3K, phosphoinositide 3-kinase; P-SMAD, phosphorylated SMAD; RBPJK, recombination signal binding protein for immunoglobulin kappa $\mathrm{J}$ region; R-SMAD, receptor-regulated SMAD; TF, transcription factor; TGF, transforming growth factor; Tmem100, transmembrane protein 100; $\operatorname{VEGF(R),~vascular~endothelial~growth~factor~(receptor).~}$ 
(Figure 1). DNA-binding cofactors strengthen the SMADDNA interaction, and interaction with coactivators and/or corepressors may affect the affinity and regulation of SMAD-responsive target genes (Figure 1). The diversity of the SMAD-interacting proteins and/or transcriptional binding partners [26] enables crosstalk between BMP and many other signaling pathways, including the Notch pathway $[28,29]$. BMP-responsive elements (BREs) typically contain a GC-rich sequence (GC-rich SMAD-binding element) and a GTCT motif that bind P-SMAD1, -5 , and -8 and SMAD4, respectively. The genes encoding inhibitors of differentiation or inhibitors of DNA binding proteins (IDs) are among the best-documented target genes for BMP-SMADs. These helix-loop-helix (HLH) proteins negatively regulate cell differentiation and stimulate cell cycle progression [30,31].

The duration and intensity of BMP signaling activity is exquisitely (auto-)regulated by several extra- and intracellular fine-tuning modulators and feedback mechanisms $[29,32]$, which help shape gradients of BMP activity in areas of uniform ligand expression. Noggin, BMP binding endothelial regulator (BMPER; cv-2 in invertebrates), BMP and activin membrane-bound inhibitor homolog (BAMBI), and inhibitory SMADs (SMAD6 and SMAD7) are examples of BMP signaling modulators expressed in ECs [33].

\section{DLL-Notch and BMPs in vascular development}

Activation of Notch by cell-bound ligands, such as DLL4 in ECs, results in proteolytic cleavage of the Notch intracellular domain (NICD) and its translocation to the nucleus, where it is recruited to target genes via interaction with the DNA-binding protein CSL/RBPJ/CBF1 [34]; Box 1; Figure 1). This complex recruits additional transcriptional activators and induces target genes, such as those encoding hairy and enhancer of split (HES) and hairy/enhancer-ofsplit-related with YRPW motif (HEY), forming the NotchCSL-HES axis. Rhythmic expression of Notch intracellular effector proteins drives many biological events (Box 2).

The DLL-Notch pathway elicits short-range, binary 'on/ off' switch mode responses between signal-sending and receiving cells in direct physical contact. This is unlike BMP morphogens that may evoke different concentrationdependent responses in a responsive tissue, even at a distance. The best-documented BMPs with functions in endothelium are BMP2, $-4,-6,-7,-9$, and $-10[33,35,36]$. BMPs can be induced locally, for example by an injured and/or hypoxic tissue to promote angiogenesis (BMP2, -4 , 6 , and -7), whereas other BMPs can circulate in high concentrations in blood (BMP4, -9 , and -10) in humans or rodents, suggesting that they play a role in maintaining normal vascular homeostasis $[33,37,38]$. Indeed, in vitro studies indicate that BMP9 inhibits angiogenesis induced by basic fibroblast growth factor 2 (bFGF) and VEGF $[15,16]$. In addition to the diverse functions of BMPs in ECs, several BMPs also regulate smooth muscle cell proliferation and inhibit their differentiation [35].

The distinct functional properties of BMPs in different vascular beds and cells derived thereof, as well as the dynamic and complex fine-tuning of the BMP pathway (reviewed in [29]), has hampered the functional

\section{Box 2. Synchronized and nonsynchronized HES1 oscillatory} gene expression

During development, Hes 1 expression oscillates in many cell types, such as fibroblasts, myoblasts, and neural stem or progenitor cells with a periodicity of approximately $2 \mathrm{~h}$ [64,97]. The rhythmic expression of Hes 1 depends on HES1 repressing its own expression through direct binding to the $\mathrm{N}$-box located in the promoter region of HES1 $[87,88]$. This negative feedback leads to the disappearance of Hes1 mRNA. HES-mediated Notch signaling regulates many dynamic processes via synchronized and nonsynchronized oscillatory gene expression. One example of a Notch-mediated dynamic process in the embryo is the rhythmic development of somites, which depends on synchronized oscillatory expression of the Notch effector gene Hes7 determining when presomitic mesoderm becomes segmented; this is called the segmentation clock [74]. High levels of Wnt and FGF signaling are required for segmentation clock activity. In mouse, a new somite pair forms every $2 \mathrm{~h}$, whereas it takes only 90 and $30 \mathrm{~min}$ in chicken and zebrafish, respectively, demonstrating that the oscillation period of the clock is species specific despite the conservation of the Notch signaling cascade and Hes oscillations. Indeed, researchers [75] recently compared mouse, chicken, and zebrafish oscillatory presomitic mesoderm transcriptomes. These networks of cyclic genes mostly involved Notch, Wnt, and FGF signaling components; however, the identity of individual cyclic genes was surprisingly different among the three species, illustrating a remarkable evolutionary plasticity of segmentation networks.

Nonsynchronous oscillatory gene expression

Hes-mediated Notch signaling has been shown to play an important role in neuronal differentiation and the maintenance of neural stem or progenitor cells $[34,98,99]$. Oscillations of HES1 levels control the expression levels of the Notch ligand DLL1 and proneural genes, such as that encoding neurogenin2 (Ngn2) [98]. Intriguingly, not all cells in the developing brain express Hes 1 in an oscillatory manner; cells in the roof plate, floor plate, and boundary regions, such as the isthmus, exhibit sustained elevated Hes 1 expression. These cells are either dormant, quiescent, or proliferate slowly, and do not give rise to any neurons, probably because high levels of Hes 1 constitutively repress the expression of proneural genes and cell cycle regulators [70]. Altogether, this illustrates that the regulation and function of HES proteins in the embryonic and adult contexts is versatile and may involve diverse mechanisms.

and mechanistic dissection of this signaling cascade in vascular development and homeostasis. However, there is evidence that BMP signaling plays (a) critical role(s) in regulating these processes based on studies in patients with rare hereditary vascular diseases that carry heterozygous mutations in genes encoding the BMP (co)-receptors endoglin, ALK1, and BMP receptor type II (BMPR2), which were discussed recently in excellent reviews [33,35,39]. In addition, vascular injury, hypertension, atherosclerosis, vascular calcification, and tumor angiogenesis have been related to aberrant production of BMPs or BMP signaling modulators $[33,35,40]$.

Different loss-of-function approaches in zebrafish and mouse models have appeared powerful in untangling the context-dependent signaling by different BMP family members (BMP2, $-4,-6$, and -7 versus BMP9 and -10 ). It was recently reported that BMP signaling regulates the initial sprouting steps from the axial vein but not from the dorsal aorta in zebrafish embryos [41]. Axial vein sprouting largely depends on BMP2-related pro-angiogenic signaling through ALK3-ALK6-BMPR2 receptor complexes and requires Disabled homolog 2 (Dab2)-mediated endocytosis of the BMP2 ligand-receptor complex [42]. Several other studies in mouse and zebrafish suggest an important role 
for ALK1-mediated BMP signaling in arterial sprouting and morphogenesis, and in the interpretation of hemodynamic forces necessary for vessel caliber control $[20,33,43-$ 46]. Importantly, overexpression of a BMP antagonist such as Noggin will interfere with venous sprouting [41], but will not antagonize BMP9, -10 and/or TGF $\beta$ induced ALK1 signaling $[47,48]$ in arterial endothelium.

The cooperation between BMP and Notch signaling is best documented in arterial endothelium. Here, we summarize recent evidence of dynamic crosstalk between Notch and BMP-SMAD-mediated signaling in the developing mouse vasculature $[20,38,49,50]$. The cooperation between BMP and Notch signaling is best documented in arterial endothelium, which is characterized by active Notch signaling and BMP9 and/or -10 induced ALK1 signaling. Synergy and subsequent antagonism of
BMP-SMAD and Notch-mediated signaling pathways have been demonstrated in EC cultures [51] and other cell types [52,53]. It has been shown in ECs that receptoractivated BMP-SMADs form a complex with NICD and potentiate expression of HES1 and HEY1 [51] (Figure 2a). Furthermore, IDs can directly interact through their HLH domain with HES1, suppressing HES1 binding to the Nbox in its own promoter (Boxes 1 and 2). Hence, IDs break the autorepression of HES1 and stabilize HES1 expression, but they do not interfere with the HES1 suppressor activity on other target genes with C-type HES1-binding sites [52] (Figure 2a). Direct binding of HES1 to the promoter region of $D L L 1$ and $D L L 4$ is reported to regulate negatively the expression of $D L L 1$ and $D L L 4$ in stem cells $[54,55]$, and overproduction of HES1 or IDs in ECs results in downregulation of DLL4 and other tip cell markers [20].

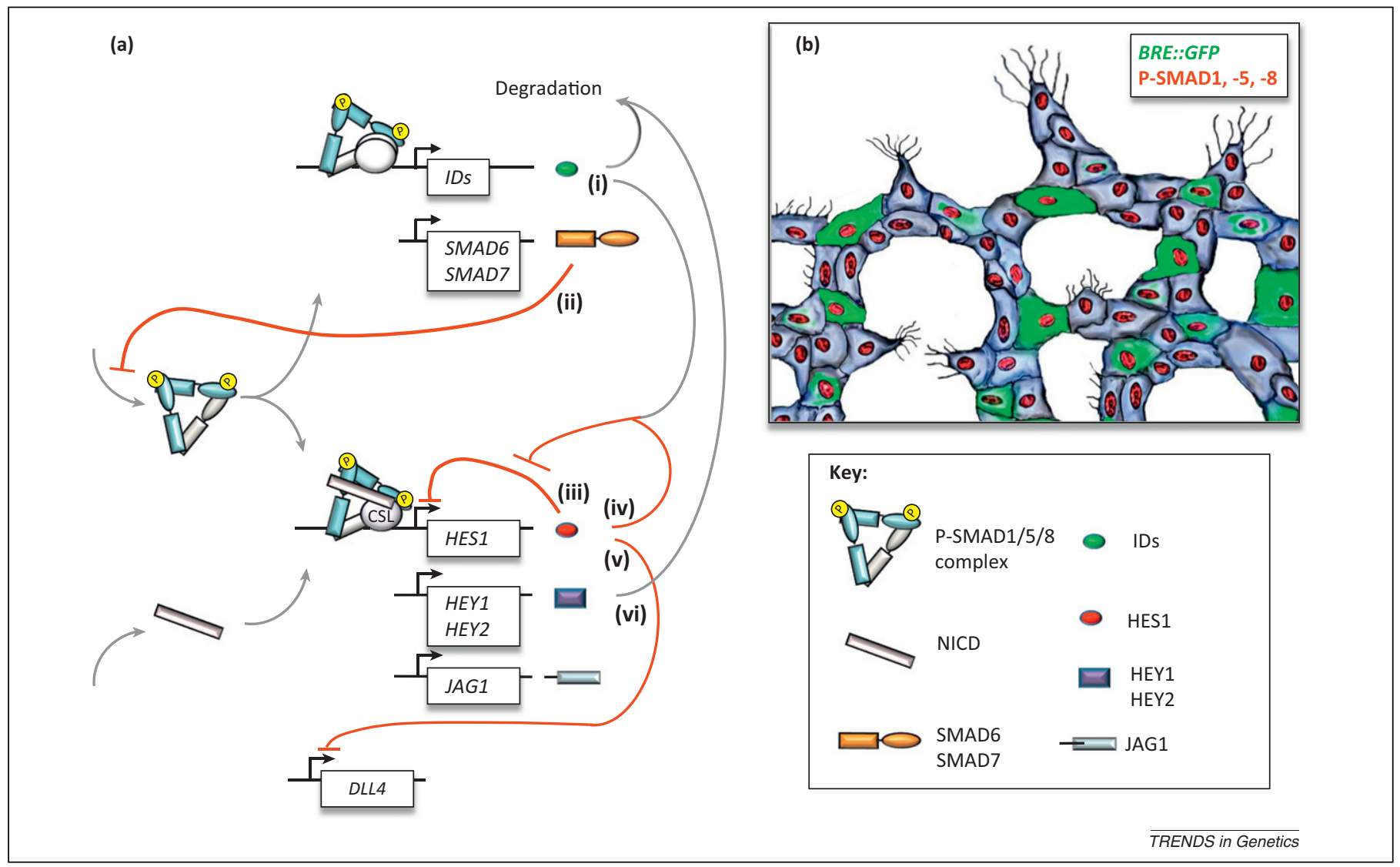

Figure 2. Crosstalk between BMP-SMAD and Notch effector drives nonsynchronous oscillatory gene expression patterns in endothelium.(a) BMP-SMAD signaling induces

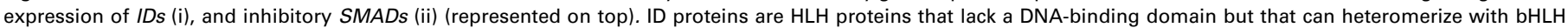
proteins, such as HES and HEY. The I-SMADs, SMAD6 and -7, attenuate activation of R-SMADS and their complex formation with co-SMAD, hence increased levels of ISMADs will result in decreased expression of NICD-independent and -dependent BMP-SMAD target genes. NICD and phosphorylated BMP-SMADs cooperatively induce target genes (bottom), including the transcriptional repressor HES1. HES1 homodimers autoinhibit their own transcription by directly binding to the N-box in the HES1 promoter (iii). This negative feedback leads to the rapid disappearance of the extremely unstable HES1 protein and allows the next round of its expression. When ID levels increase, HES1 autoinhibition is released through ID:HES1 protein-protein interactions (iv) that interfere with HES1 binding to N-box sites (Box 1), but not to C-box sites in promoters of HES1 target genes [51]. This results in increased HES1 protein levels and subsequent temporal repression of $D L L 4$ (v) [54,55], which reduces Notch signaling in neighboring cells. The composition of the HES1 dimer that represses DLL4 is unclear. Other NICD-SMAD targets include HEY1 and -2 and JAG1. HEY1 and -2 are also bHLH proteins that can interact with IDs and destabilize them (vi) [20,51], which in turn impacts the HES1 and DLL4 levels. Furthermore, HEY1 and -2 have been shown to be induced by BMP9 in a Notch/CSL-independent fashion [49], and HEY1 can also interact with HES proteins, which may underlie the unstable nature of HES1 (not depicted for simplicity). The HEY protein interactions therefore turn the initial synergy of BMP-Notch signaling into an antagonism. (b) Schematic representation of the differential localization of P-SMAD1, -5, and -8 and BRE::gfp in angiogenic endothelium in E9.5 mouse embryo based on [20]. Nuclear P-SMAD1, -5, and -8 can be detected throughout

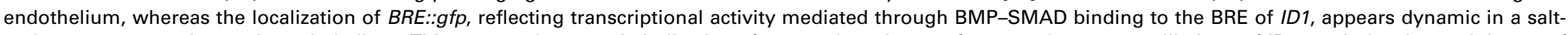
and-pepper pattern in nontip endothelium. This scattered pattern is indicative of a snapshot picture of nonsynchronous oscillations of ID protein levels, reminiscent of nonsynchronous oscillations of HES1 in neural stem cells and/or progenitors [86]. This pattern in angiogenic endothelium is suggestive of the coupled transcriptional feedback loops between BMP and Notch pathways, as reported in EC culture [51]. Abbreviations: BMP, bone morphogenetic protein; BRE, BMP-responsive elements; CSL, CBF1, Su(H) and LAG-1; co-SMAD, common-mediator SMAD; DLL4, delta-like 4; EC, endothelial cell; HES1, hairy and enhancer of split 1; HEY, hairy/enhancer-of-split related with YRPW motif; (b)HLH, (basic) helix-loop-helix; I-SMAD, inhibitory SMAD; ID, inhibitors of differentiation or inhibitor of DNA-binding proteins; JAG1, jagged 1; NICD, Notch intracellular domain; P-SMAD, phosphorylated SMAD; R-SMAD, receptor-regulated SMAD. 
HEY1 antagonizes BMP/ID1-induced migration of ECs by promoting ID protein degradation, and hence Notch antagonizes BMP signaling [51]. Recently, ChIP and ChIP-Seq studies in BMP-stimulated ECs showed that Notch target genes, including $H E Y 1$ and those encoding Ncadherin (CDH1), and jagged 1 (JAG1) are direct targets of BMP-SMADs [50,56] (Figure 2a), which further substantiates the interdependence of these pathways.

Given the redundant as well as nonredundant, and even opposing functions of distinct BMPs in ECs [35,57], in vivo studies have increasingly focused on investigating BMP receptors or BMP-SMAD function. To monitor in vivo spatiotemporally active BMP-SMAD signaling in endothelium, nuclear localization of P-SMAD1, -5 , and -8 is commonly used. However, this localization may not exclusively report BMP signaling. Indeed, TGF $\beta$, which binds other receptors and regulates target genes distinct from BMP targets through activation of SMAD2 and -3 , has been shown to bind weakly the EC-specific type I receptor for BMP9 and -10 (ALK1) in concert with the TGF $\beta$ type II receptor, and induce phosphorylation of SMAD1, -5 , and -8 [58]. Likewise, the recently described BMP-SMAD activity reporters (based on SMAD1, -5, and -8-SMAD4 binding elements from $I d 1$ or Xenopus Vent2) in mouse and zebrafish embryos [59-63] may also reflect signaling activity from non-BMPs, whereas BMP non-SMAD signaling will remain unnoticed using this read-out. Nonetheless, the combination of monitoring P-SMAD1, -5 , and -8 and reporter activity was instrumental in revealing nonsynchronized BMP signaling in angiogenic endothelium and the relevance of BMP-SMAD signaling for tip and stalk cell selection and function. Indeed, nuclear localization of PSMAD1, -5 , and -8 was detected throughout the vasculature of an E9.5 developing mouse embryo, whereas the activity of a BMP-SMAD signaling reporter based on the BRE of IdI and the distribution of ID proteins themselves was restricted to nascent stalk cells flanking early tip cells and scattered ECs in nonsprouting endothelium, but was not detected in tip cells (Figure 2b) [20]. This differential localization pattern suggests that P-SMAD1, -5 , and -8 activate distinct target genes in tip and stalk cells and implicates non-ID-dependent BMP-SMAD activities in the regulation of tip cell polarity and directed cell migration [20]. The scattered ID localization pattern could reflect a snapshot of nonsynchronized oscillatory gene expression, such as the nonsynchronized oscillation of Hes1 protein in neural precursors in E14.5 mouse embryos (Box 2) [64].

The scattered ID localization in ECs in E9.5 embryos suggests a dynamic role for IDs in Notch-mediated stalk cell competence and specification, which was further supported by chimeric competition experiments with control and SMAD1, and -5-knockdown ECs, or ECs that overproduce IDs [20]. Importantly, EC-specific inactivation of Smad1 and Smad5 causes a hypersprouting defect reminiscent of impaired Notch-mediated signaling in mouse embryos [20]. Yet, IDs play only a permissive role in Notch-HES1 signaling and stalk cell competence, because inhibition of Notch signaling results in increased tip cell formation [65], whereas the ID distribution expands in this condition [20], indicating that ID proteins do not determine the stalk cell phenotype per se. Stabilization of the ID localization likely results from reduced HEY levels and concomitant reduced ID degradation [51,66] (Figure 2a).

\section{BMP-SMADS and IDs as essential components of the HES1 molecular oscillator}

The mutual interdependence between Notch and BMPSMAD cascades in ECs may result in dynamic amplification and subsequent inhibition of downstream targets of either cascade [51,67] (Figure 2a). It was proposed that the resulting nonsynchronized oscillations of BMP-SMAD and Notch effectors provide individual ECs stalk and tip cell competence. In the absence of pro-angiogenic stimuli, ECs become periodically competent to each phase [20] (Figure 3a). The intervals between each competence phase ensure that a sufficient pool of permissive nonsprouting ECs is maintained when a group of cells becomes exposed to pro-angiogenic stimuli. Only ECs that are in their tip cell-competence phase, with sufficient low levels of the transcriptional repressor HES1 and corresponding high levels of DLL4, will instantly respond to pro-angiogenic stimuli and become further enriched in DLL4, whereas the prolonged absence or the lower levels of HES1 allow enrichment of pro-tip cell genes (Figure $3 b$ ). The immediate neighbors of the newly formed tip cell with the highest ID levels will elicit the greatest Notch output, resulting in an out-of-phase HES1 oscillation (Figure 3c). The increased HES1 levels and/or the prolonged HES1 presence allow efficient repression of not only $D L L 4$, but also other pro-tip cell genes, and activation of the stalk cell program. A sharp boundary between the tip cell and stalk cell is thus established. However, increasing HEY levels in the stalk cell cause an amplified ID degradation (Figure 3c), which gradually leads to the restoration of the oscillatory network because the high instability of the HES1 protein will progressively lead again to accumulation of DLL4 levels, and lateral inhibition of the tip cell.

This nonsynchronized oscillation model of BMP-SMAD and Notch effectors was fueled by the hypersprouting phenotype in mouse embryos with an EC-specific inactivation of Smad1 and Smad5 and time-course transcriptional analyses in ECs with gain-of-function of IDs (or HES1 or NICD) or loss-of-function of BMP-SMADs [20]. Furthermore, waves of endogenous ID-HES1 and ID-HEY2 protein complexes were revealed by proximity ligation assays [20]. Increased numbers of tip cell-like cells in the ECspecific Smad1;Smad5 mutant embryos are associated with absence of IDs, whereas DLL4 and nuclear NICD localization patterns are expanded and no longer complementary. However, the expanded localization of NICD fails to induce the stalk cell behavior, which is likely due to an inability to accumulate sufficient HES1 levels to repress $D L L 4$ and tip cell-enriched genes in the stalk cell compartment [20].

The mutual interdependence of BMP-SMAD and Notch pathways was further supported by two independent studies $[38,49]$. One reported that blocking of ALK1 signaling during postnatal development in mice leads to retinal hypersprouting and arteriovenous malformations [49]. The authors showed that BMP9 and -10-induced ALK1dependent signaling synergizes through SMADs with Notch signaling and co-induces expression of the Notch 


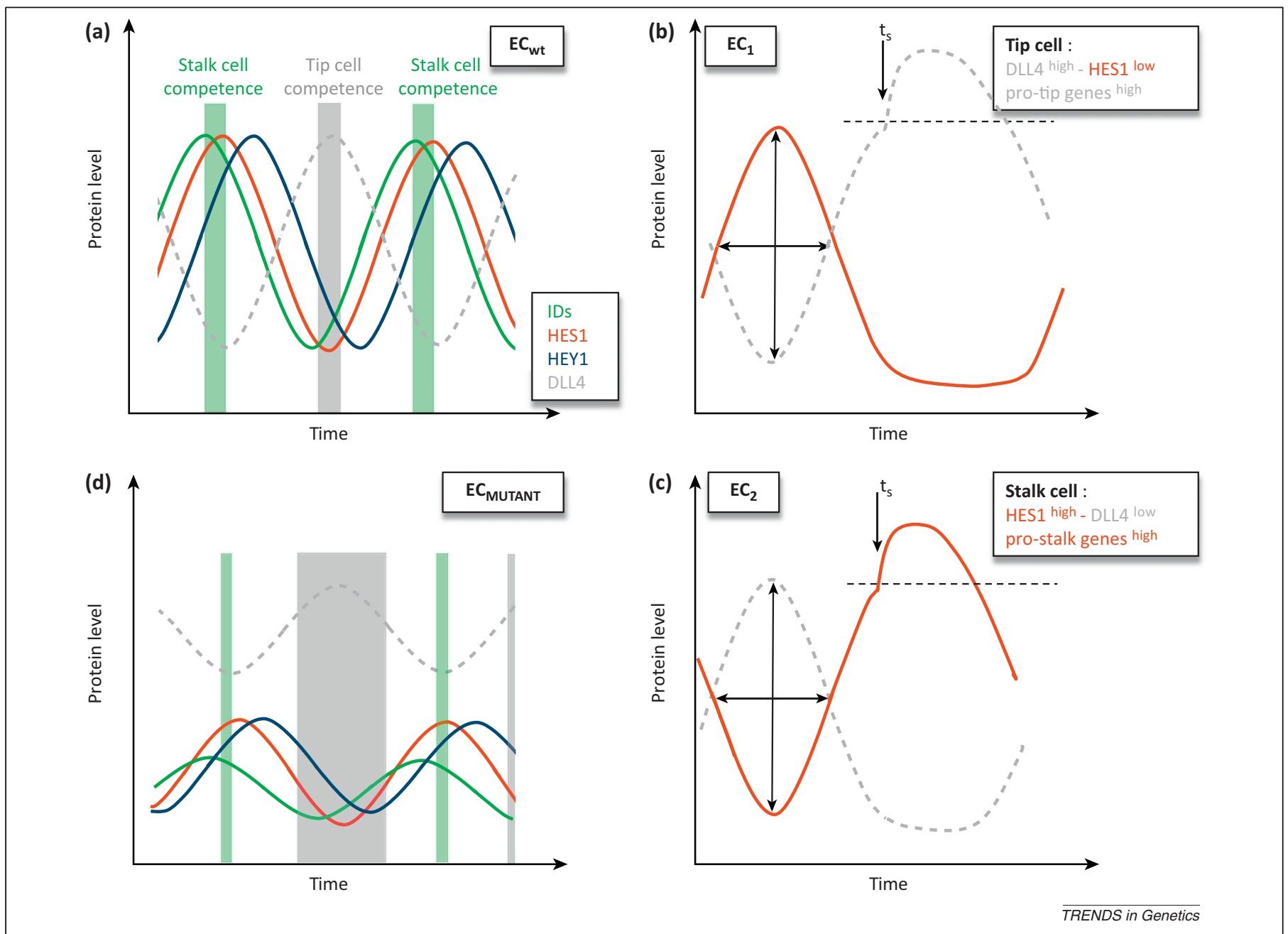

Figure 3. Nonsynchronous oscillations of BMP-SMAD and Notch effectors dynamically regulate EC competence. (a) The synergy and antagonism between BMP-Notch

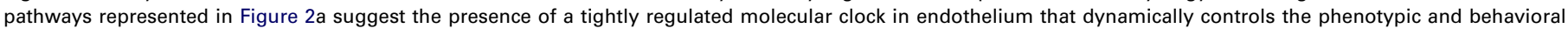
response capacities of single ECs. In the proposed model, the interplay between ultradian oscillations of BMP-SMAD and Notch effectors determines periodically the tip versus stalk cell competence in the absence of an angiogenic stimulus. The competence of a cell will be determined by its relative HES1-DLL4 levels. The cell can be triggered to become a tip cell at intervals of low HES1 levels and hence high DLL4 levels, whereas high levels of ID and HES1 provide a window in which the same EC can be induced to become a stalk cell. Nonsynchronous oscillations in a population of cells permit differential responses when exposed to the same angiogenic stimulus. In all models, the protein levels are arbitrarily drawn and oscillations for different effectors may have different amplitudes. (b,c) When a population of permissive ECs with stochastic, nonsynchronous oscillatory levels of BMP-SMAD (ID) and BMP-SMAD-NICD (HES1 and HEY1 and -2) effector proteins is exposed to a pro-angiogenic stimulus

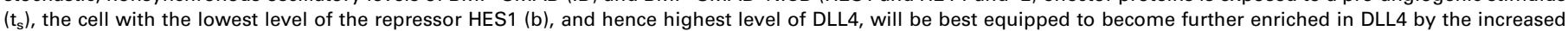
VEGFR-2 and -3 signaling. Hence, this cell $\left(\mathrm{EC}_{1}\right)$ is selected as the tip cell. Prolonged absence of HES1 allows sufficient enrichment of DLL4 and induction of a repertoire of pro-tip cell genes. The tip cell will establish a temporal boundary with its neighboring cells. (c) Of all the flanking cells of the tip cell, the cell with the highest levels of ID $\left(E C_{2}\right)$ will yield the highest increase in Notch-HES1 output (lateral inhibition) and will hence acquire the stalk cell phenotype. A higher Notch output results in higher and/or prolonged HES1 and HEY1 and -2 levels, causing prolonged DLL4 and pro-tip cell gene repression necessary to activate the stalk cell behavior. Increased HEY1 and - 2 levels subsequently trigger the abrupt degradation of ID proteins, which will contribute to the stalk cell progressively raising DLL4 levels again and becoming once more a permissive EC that can eventually shuffle to the tip cell position. Indeed, the adjacent tip cell (b) will progressively undergo more DLL4-Notch signaling and HES1 will become induced; at this stage, the tip cell position can be taken over or is lost upon anastomosis. Experimental data are still lacking that substantiate whether HES1 levels (i) increase and raise the amplitude of the oscillation; (ii) prolong and result in lengthened wave periods; or (iii) a combination of both happens during tip and stalk cell selection. Here, we postulate a combination of increased amplitude and wave period. In this model, the ID levels (which stabilize HES1 protein levels) make ECs dynamically permissive to the stalk cell phenotype, whereas the HES1 and DLL4 levels will control the EC phenotype at any time. (d) Deficiency in BMP signaling component(s) resulting in severely reduced ID levels in endothelium can be expected to impact all other components of the oscillatory network and the resulting EC competence. The interval of tip cell competence will become expanded, with a consequent failure to establish a sharp tip-stalk cell boundary due to insufficiently high differential HES1 protein levels between the early tip and the neighboring cells. Furthermore, the pool of nonsprouting ECs will decrease. This hypothetical model is based on gene transcription studies in

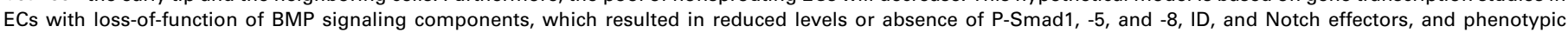
observations in mice with (endothelial-specific) deficiency in SMAD1 and -5, ALK1, or BMP9 and -10 [20,38,49]. These mouse models all developed vascular hypersprouting defects with excessive tip cell-like cells. Abbreviations: ALK1, activin receptor-like kinase 1; BMP, bone morphogenetic protein; DLL4, Disc-like ligand 4; EC, endothelial cell;

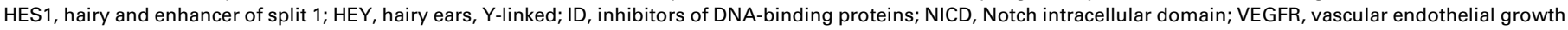
factor receptor.

targets $H E Y 1$ and $H E Y 2$, thereby repressing VEGF signaling and tip cell formation. Mosaic bead-sprouting assays with ALK1-, SMAD4-, or HEY2-knockdown ECs, showed their enrichment at the tip cell position, which was in line with similar experiments using SMAD1 and -5 knockdown ECs [20]. Additionally, the BMP9 and -10 study showed that BMP-ALK1-induced BMP-SMAD signaling can induce $H E Y 1$ and $H E Y 2$ independently of canonical Notch activation [49].

A second group showed that mice deficient in BMP9 (the major circulating ligand thought to activate ALK1) are surprisingly viable with normal retinal vasculature [38]. 
However, the additional injection of neutralizing antibodies against BMP10 resulted in impaired postnatal vascular remodeling of the retina, illustrating that BMP10 can substitute for BMP9. Furthermore, BMP9 or BMP10 signaling results in transient increased expression of Notch effectors (JAG1, HES1 and HEY1 and -2), brief induction of $D L L 4$ with subsequent $D L L 4$ suppression below baseline levels, and decreased expression of the gene encoding apelin $(A P L N)$ in ECs in culture, compatible with a loss of tip cell behavior.

Collectively, these results identify BMP-SMADs and IDs as crucial dynamic regulators of the HES-HEY molecular oscillator, and the BMP9 and $-10 \rightarrow \mathrm{ALK} 1 \rightarrow \mathrm{BMP}-$ SMAD pathway as an integral player in priming vessel plasticity. We propose that significantly reduced levels of IDs in these mutants will affect the other components of the oscillatory network, resulting in expansion of the tip cell competence phase and a hypersprouting defect (Figure 3d). This model is in agreement with the hypersprouting defect observed in Dll4 heterozygous mice [65], which results from insufficient Notch signaling in the signal receiving cells (the tip neighbors) and concomitant insufficient high and/or long (out-of-phase) increase in HES1 levels to trigger a robust stalk cell behavior. Decreased HEY-mediated degradation of IDs could lead to more widespread ID localization in such conditions.

\section{Concluding remarks}

Dynamic nonsynchronized oscillatory networks of BMPSMAD and Notch intracellular effector proteins may establish rapid and robust tip-stalk cell selection. This model is compatible with tip and stalk cells being in continuous flux, the shuffling of tip and stalk cell position, and Notchmediated macrophage-assisted anastomosis $[68,69]$. Notably, this cross-signaling also leads to maintenance of a dynamic pool of permissive, nonsprouting ECs. Similar to the cells in the roof plate and floor plate of the neural tube and boundary regions in the central nervous system, which express Hes1 in a sustained manner [70], HES1 levels are likely to be more stable and persist longer in quiescent or slowly proliferating endothelium (phalanx cells), but how this is regulated remains elusive.

Waves of SMAD1, -5 , and -8 phosphorylation are reported to result from the (periodic) induction of inhibitory SMADs in fibroblasts [71], a feedback loop that can also contribute to BMP and/or Notch effector oscillations in ECs (Figures 1a and 2a), but other fine-tuning mechanisms may contribute equally to periodic regulation of SMAD1, -5 , and -8 phosphorylation (reviewed in [29]). The relative protein levels of phosphorylated BMP-SMADs, ID, I-SMAD, HES, and HEY, their distinct protein:protein and protein:promoter binding affinities, and their respective protein stabilities may result in oscillatory waves of different magnitude that dynamically determine differential target gene regulation and EC behavior.

Oscillatory levels of IDs also likely (in)directly affect oscillators other than HES1. It is noteworthy that p53 levels also oscillate [72] and that IDs act in ECs as proproliferative factors that antagonize p53-mediated cell cycle regulation [73]. Conversely, hedgehog, FGF, Wnt, and Janus kinase-signal transducers and activators of transcription (JAK-STAT) signaling have been shown to coregulate oscillations of HES1 activity in other cell types [71,74,75] and may also impinge on BMP- and Notchcoregulated HES1 oscillations in ECs. Integrin receptors for basement membrane proteins likely also function as nodes in BMP- and Notch-coregulated tip versus stalk cell selection. Integrins are differentially regulated by BMPs and TGF $\beta$ and they play a crucial role in controlling EC proliferation and migration. Recent reports have shown that specific laminin and integrin-induced signaling regulates sprouting angiogenesis by inducing DLL4-Notch signaling [76,77]. Furthermore, fibronectin and $\alpha 5 \beta 1$ integrin increase TGF- $\beta 1$ and BMP9-induced Smad1, -5, and -8 phosphorylation in an endoglin- and ALK1-dependent manner [78], in turn affecting Notch signaling.

Although the overall emerging picture of Notch and BMP-SMAD cooperation and the concept of nonsynchronized oscillatory gene regulation regulating EC competence and behavior are taking shape, important pieces of this puzzle are still missing. The current review has zoomed in on the link between Notch and BMP9 and -10 induced Smad1 and -5 mediated ALK1 signaling in ECs during (arterial) sprouting angiogenesis, but has disregarded thus far (for reasons of simplicity) the link between Notch and Smad 2 and -3 mediated TGF $\beta$ signaling, or the mural cell component of the vessel wall [48]. However, receptor-activated SMAD2, -3- and SMAD1, -5, -8-SMAD4 complexes both stabilize cerebrovascular EC-pericyte interactions by regulating expression of the gene encoding $\mathrm{N}$-cadherin $(\mathrm{CDH} 1)$ in a SMAD- and NICD-dependent fashion [50]. Furthermore, the intracellular domain (ICD) of DLL1 has been shown to mediate TGF $\beta$ signaling through binding to SMAD2 and -3 and enhancing SMADdependent transcription, indicative of an important role for bidirectional Notch-DLL signaling in neural stem cells [79-81]. Although DLL4-ICD interactions with BMPSMADs have not yet been reported, it is tempting to postulate a similar role for DLL4-ICD and BMP-SMADs that may function independently of IDs in tip cells.

Little is known about how specific BMP ligands and Notch-BMP-SMAD cooperation could act as organ-specific signals regulating particular steps of angiogenesis in different vascular beds. This is partly because the BMP receptor distribution is poorly documented in different types of EC in vivo. It is however tempting to speculate that Notch and BMP-SMAD effectors may regulate oscillatory gene networks in the lymphatic system, because blocking ALK1 signaling results in severe defects in mouse neonates in lymphatic development in multiple organs [82], and DLL4-Notch signaling controls lymphatic sprouting and direct stalk cell specification in lymphatic ECs [8385]. Exploring this hypothesis and continuing to investigate the molecular mechanisms that coregulate vessel plasticity and patterning in different vascular beds may result in refinement of combinatorial anti-angiogenesis and vessel normalization therapies.

\section{Acknowledgments}

We thank past and present team members for stimulating discussions and support. We apologize to authors whose relevant work was not cited because of space restrictions. This work was supported by VIB, OT-09/ 053, and GOA-11/012 from the KU Leuven Research Council. 


\section{References}

1 Geudens, I. and Gerhardt, H. (2011) Coordinating cell behaviour during blood vessel formation. Development 138, 4569-4583

2 Strasser, G.A. et al. (2010) Microarray analysis of retinal endothelial tip cells identifies CXCR4 as a mediator of tip cell morphology and branching. Blood 115, 5102-5110

3 del Toro, R. et al. (2010) Identification and functional analysis of endothelial tip cell-enriched genes. Blood 116, 4025-4033

4 Tammela, T. et al. (2011) VEGFR-3 controls tip to stalk conversion at vessel fusion sites by reinforcing Notch signalling. Nat. Cell Biol. 13, 1202-1213

5 Benedito, R. et al. (2012) Notch-dependent VEGFR3 upregulation allows angiogenesis without VEGF-VEGFR2 signalling. Nature 484, 110-114

6 Jakobsson, L. et al. (2010) Endothelial cells dynamically compete for the tip cell position during angiogenic sprouting. Nat. Cell Biol. 12 , 943-953

7 Blanco, R. and Gerhardt, H. (2012) VEGF and Notch in tip and stalk cell selection. Cold Spring Harb. Perspect. Med. 1, 133-136

8 Eichmann, A. and Simons, M. (2012) VEGF signaling inside vascular endothelial cells and beyond. Curr. Opin. Cell Biol. 24, 188-193

9 Tung, J.J. et al. (2012) Tips, stalks, tubes: Notch-mediated cell fate determination and mechanisms of tubulogenesis during angiogenesis. Cold Spring Harb. Perspect. Med. 2, a006601

10 Miyazono, K. et al. (2000) TGF-beta signaling by Smad proteins. Adv. Immunol. 75, 115-157

11 Urist, M.R. (1965) Bone: formation by autoinduction. Science 150, 893-899

12 Wozney, J.M. et al. (1988) Novel regulators of bone formation: molecular clones and activities. Science 242, 1528-1534

13 Wagner, D.O. et al. (2010) BMPs: from bone to body morphogenetic proteins. Sci. Signal. 2, 3

14 Townson, S.A. et al. (2012) Specificity and structure of a high affinity activin receptor-like kinase 1 (ALK1) signaling complex. J. Biol. Chem. 287, 27313-27325

15 David, L. et al. (2007) Identification of BMP9 and BMP10 as functional activators of the orphan activin receptor-like kinase 1 (ALK1) in endothelial cells. Blood 109, 1953-1961

16 Scharpfenecker, M. et al. (2007) BMP-9 signals via ALK1 and inhibits bFGF-induced endothelial cell proliferation and VEGF-stimulated angiogenesis. J. Cell Sci. 120, 964-972

17 Mahlawat, P. et al. (2012) Structure of the Alk1 extracellular domain and characterization of its bone morphogenetic protein (BMP) binding properties. Biochemistry 51, 6328-6341

18 Pangas, S.A. et al. (2008) Conditional deletion of Smad1 and Smad5 in somatic cells of male and female gonads leads to metastatic tumor development in mice. Mol. Cell. Biol. 28, 248-257

19 Umans, L. et al. (2007) Inactivation of Smad5 in endothelial cells and smooth muscle cells demonstrates that Smad5 is required for cardiac homeostasis. Am. J. Pathol. 170, 1460-1472

20 Moya, I.M. et al. (2012) Stalk cell phenotype depends on integration of Notch and Smad1/5 signaling cascades. Dev. Cell 22, 501-514

21 Arnold, S.J. et al. (2006) Dose-dependent Smad1, Smad5 and Smad8 signaling in the early mouse embryo. Dev. Biol. 296, 104-118

22 Drake, K.M. et al. (2011) Altered MicroRNA processing in heritable pulmonary arterial hypertension: an important role for Smad-8. Am. J. Respir. Crit. Care Med. 184, 1400-1408

23 Shintani, M. et al. (2009) A new nonsense mutation of SMAD8 associated with pulmonary arterial hypertension. J. Med. Genet. 46, 331-337

24 Huang, Z. et al. (2009) Defective pulmonary vascular remodeling in Smad8 mutant mice. Hum. Mol. Genet. 18, 2791-2801

$25 \mathrm{Wu}, \mathrm{M} . Y$. and Hill, C.S. (2009) Tgf-beta superfamily signaling in embryonic development and homeostasis. Dev. Cell 16, 329-343

26 Conidi, A. et al. (2011) Few Smad proteins and many Smad-interacting proteins yield multiple functions and action modes in TGFbeta/BMP signaling in vivo. Cytokine Growth Factor Rev. 22, 287-300

27 Morikawa, M. et al. (2012) Genome-wide mechanisms of Smad binding. Oncogene http://dx.doi.org/10.1038/onc.2012.191

28 Guo, X. and Wang, X.F. (2009) Signaling cross-talk between TGF-beta/ BMP and other pathways. Cell Res. 19, 71-88

29 Ramel, M.C. and Hill, C.S. (2012) Spatial regulation of BMP activity. FEBS Lett. 586, 1929-1941
30 Norton, J.D. and Atherton, G.T. (1998) Coupling of cell growth control and apoptosis functions of Id proteins. Mol. Cell. Biol. 18, 2371-2381

31 Zebedee, Z. and Hara, E. (2001) Id proteins in cell cycle control and cellular senescence. Oncogene 20, 8317-8325

32 Nickel, J. et al. (2009) Intricacies of BMP receptor assembly. Cytokine Growth Factor Rev. 20, 367-377

33 Cai, J.et al. (2012) BMP signaling in vascular diseases. FEBS Lett. 586 , 1993-2002

34 Kopan, R. and Ilagan, M.X. (2009) The canonical Notch signaling pathway: unfolding the activation mechanism. Cell 137, 216-233

35 Lowery, J.W. and de Caestecker, M.P. (2010) BMP signaling in vascular development and disease. Cytokine Growth Factor Rev. 21, 287-298

36 David, L. et al. (2009) Emerging role of bone morphogenetic proteins in angiogenesis. Cytokine Growth Factor Rev. 20, 203-212

37 Herrera, B. and Inman, G.J. (2009) A rapid and sensitive bioassay for the simultaneous measurement of multiple bone morphogenetic proteins. Identification and quantification of BMP4, BMP6 and BMP9 in bovine and human serum. BMC Cell Biol. 10, 20

38 Ricard, N. et al. (2012) BMP9 and BMP10 are critical for postnatal retinal vascular remodeling. Blood 119, 6162-6171

39 Koumakis, E. et al. (2012) TGFbeta receptor gene variants in systemic sclerosis-related pulmonary arterial hypertension: results from a multicentre EUSTAR study of European Caucasian patients. Ann. Rheum. Dis. 71, 1900-1903

40 Mahmoud, M. et al. (2011) Angiogenesis regulation by TGFbeta signalling: clues from an inherited vascular disease. Biochem. Soc. Trans. 39, 1659-1666

41 Wiley, D.M. et al. (2011) Distinct signalling pathways regulate sprouting angiogenesis from the dorsal aorta and the axial vein. Nat. Cell Biol. 13, 686-692

$42 \mathrm{Kim}$, J.D. et al. (2012) Context-dependent proangiogenic function of bone morphogenetic protein signaling is mediated by disabled homolog 2. Dev. Cell 23, 441-448

43 Mahmoud, M. et al. (2009) Endoglin and activin receptor-like-kinase 1 are co-expressed in the distal vessels of the lung: implications for two familial vascular dysplasias, HHT and PAH. Lab. Invest. 89, 15-25

44 Somekawa, S. et al. (2012) Tmem100, an ALK1 receptor signalingdependent gene essential for arterial endothelium differentiation and vascular morphogenesis. Proc. Natl. Acad. Sci. U.S.A. 109, 1206412069

45 Roman, B.L. et al. (2002) Disruption of acvrl1 increases endothelial cell number in zebrafish cranial vessels. Development 129, 3009-3019

46 Corti, P. et al. (2011) Interaction between alk1 and blood flow in the development of arteriovenous malformations. Development 138, 1573-1582

47 Seemann, P. et al. (2009) Mutations in GDF5 reveal a key residue mediating BMP inhibition by NOGGIN. PLoS Genet. 5, e1000747

48 Pardali, E. et al. (2010) Signaling by members of the TGF-beta family in vascular morphogenesis and disease. Trends Cell Biol. 20, 556-567

49 Larrivee, B. et al. (2012) ALK1 signaling inhibits angiogenesis by cooperating with the Notch pathway. Dev. Cell 22, 489-500

$50 \mathrm{Li}$, F. et al. (2011) Endothelial Smad4 maintains cerebrovascular integrity by activating N-cadherin through cooperation with Notch. Dev. Cell 20, 291-302

51 Itoh, F. et al. (2004) Synergy and antagonism between Notch and BMP receptor signaling pathways in endothelial cells. EMBO J. 23, 541-551

52 Bai, G. et al. (2007) Id sustains Hes1 expression to inhibit precocious neurogenesis by releasing negative autoregulation of Hes1. Dev. Cell 13, 283-297

53 Quillien, A. et al. (2011) BMP signaling orchestrates photoreceptor specification in the zebrafish pineal gland in collab oration with Notch. Development 138, 2293-2302

54 Kobayashi, T. et al. (2009) The cyclic gene Hes1 contributes to diverse differentiation responses of embryonic stem cells. Genes Dev. 23, 1870 1875

55 Kobayashi, T. and Kageyama, R. (2010) Hes1 regulates embryonic stem cell differentiation by suppressing Notch signaling. Genes Cells 15, 689-698

56 Morikawa, M. et al. (2011) ChIP-seq reveals cell type-specific binding patterns of BMP-specific Smads and a novel binding motif. Nucleic Acids Res. 39, 8712-8727 
57 Anderson, L. et al. (2010) Bmp2 and Bmp4 exert opposing effects in hypoxic pulmonary hypertension. Am. J. Physiol. 298, R833-R842

58 Orlova, V.V. et al. (2011) Controlling angiogenesis by two unique TGF-beta type I receptor signaling pathways. Histol. Histopathol. 26, $1219-1230$

59 Monteiro, R.M. et al. (2004) Spatio-temporal activation of Smad1 and Smad5 in vivo: monitoring transcriptional activity of Smad proteins. $J$. Cell Sci. 117, 4653-4663

60 Monteiro, R.M. et al. (2008) Real time monitoring of BMP Smads transcriptional activity during mouse development. Genesis $46,335-346$

61 Collery, R.F. and Link, B.A. (2011) Dynamic smad-mediated BMP signaling revealed through transgenic zebrafish. Dev. Dyn. 240, $712-722$

62 Laux, D.W. et al. (2011) Dynamic analysis of BMP-responsive smad activity in live zebrafish embryos. Dev. Dyn. 240, 682-694

63 Javier, A.L. et al. (2012) Bmp indicator mice reveal dynamic regulation of transcriptional response. PLoS ONE 7, e42566

64 Shimojo, H. et al. (2011) Dynamic expression of Notch signaling genes in neural stem/progenitor cells. Front. Neurosci. 5, 78

65 Hellstrom, M. et al. (2007) Dll4 signalling through Notch1 regulates formation of tip cells during angiogenesis. Nature 445, 776-780

66 Henderson, A.M. et al. (2001) The basic helix-loop-helix transcription factor HESR1 regulates endothelial cell tube formation. J. Biol. Chem. $276,6169-6176$

67 Holderfield, M.T. and Hughes, C.C. (2008) Crosstalk between vascular endothelial growth factor, Notch, and transforming growth factor-beta in vascular morphogenesis. Circ. Res. 102, 637-652

68 Fantin, A. et al. (2010) Tissue macrophages act as cellular chaperones for vascular anastomosis downstream of VEGF-mediated endothelial tip cell induction. Blood 116, 829-840

69 Outtz, H.H. et al. (2011) Notch1 controls macrophage recruitment and Notch signaling is activated at sites of endothelial cell anastomosis during retinal angiogenesis in mice. Blood 118, 3436-3439

70 Baek, J.H. et al. (2006) Persistent and high levels of Hes1 expression regulate boundary formation in the developing central nervous system. Development 133, 2467-2476

71 Yoshiura, S. et al. (2007) Ultradian oscillations of Stat, Smad, and Hes1 expression in response to serum. Proc. Natl. Acad. Sci. U.S.A. 104, 11292-11297

72 Mengel, B. et al. (2010) Modeling oscillatory control in NF-kappaB, p53 and Wnt signaling. Curr. Opin. Genet. Dev. 20, 656-664

73 Qiu, J. et al. (2011) Id1-induced inhibition of p53 facilitates endothelial cell migration and tube formation by regulating the expression of beta1-integrin. Mol. Cell. Biochem. 357, 125-133

74 Pourquie, O. (2011) Vertebrate segmentation: from cyclic gene networks to scoliosis. Cell 145, 650-663

$75 \mathrm{Krol}$, A.J. et al. (2011) Evolutionary plasticity of segmentation clock networks. Development 138, 2783-2792

76 Stenzel, D. et al. (2011) Endothelial basement membrane limits tip cell formation by inducing Dll4/Notch signalling in vivo. EMBO Rep. 12, $1135-1143$

77 Estrach, S. et al. (2011) Laminin-binding integrins induce Dll4 expression and Notch signaling in endothelial cells. Circ. Res. 109, $172-182$

78 Tian, H. et al. (2012) Endoglin mediates fibronectin/alpha5beta1 integrin and TGF-beta pathway crosstalk in endothelial cells. EMBO J. 31, 3885-3900
79 Hiratochi, M. et al. (2007) The Delta intracellular domain mediates TGF-beta/Activin signaling through binding to Smads and has an important bi-directional function in the Notch-Delta signaling pathway. Nucleic Acids Res. 35, 912-922

80 Bordonaro, M. et al. (2011) The Notch ligand Delta-like 1 integrates inputs from TGFbeta/Activin and Wnt pathways. Exp. Cell Res. 317, $1368-1381$

$81 \mathrm{Fu}$, Y. et al. (2009) Differential regulation of transforming growth factor beta signaling pathways by Notch in human endothelial cells. J. Biol. Chem. 284, 19452-19462

82 Niessen, K. et al. (2010) ALK1 signaling regulates early postnatal lymphatic vessel development. Blood 115, 1654-1661

83 Niessen, K. et al. (2011) The Notch1-Dll4 signaling pathway regulates mouse postnatal lymphatic development. Blood 118, 1989-1997

84 Geudens, I. et al. (2010) Role of delta-like-4/Notch in the formation and wiring of the lymphatic network in zebrafish. Arterioscler. Thromb. Vasc. Biol. 30, 1695-1702

85 Zheng, W. et al. (2011) Notch restricts lymphatic vessel sprouting induced by vascular endothelial growth factor. Blood 118, 1154-1162

86 Kageyama, R. et al. (2007) Oscillator mechanism of Notch pathway in the segmentation clock. Dev. Dyn. 236, 1403-1409

87 Kageyama, R. et al. (2007) The Hes gene family: repressors and oscillators that orchestrate embryogenesis. Development 134, $1243-1251$

88 Grogan, S.P. et al. (2008) Repression of chondrogenesis through binding of Notch signaling proteins HES-1 and HEY-1 to N-box domains in the COL2A1 enhancer site. Arthritis Rheum. 58, 27542763

89 Kobayashi, T. and Kageyama, R. (2010) Hes1 oscillation: making variable choices for stem cell differentiation. Cell Cycle 9, 207-208

90 Upton, P.D. et al. (2009) Bone morphogenetic protein (BMP) and activin type II receptors balance BMP9 signals mediated by activin receptor-like kinase-1 in human pulmonary artery endothelial cells. $J$. Biol. Chem. 284, 15794-15804

91 Miyazono, K. et al. (2005) BMP receptor signaling: transcriptional targets, regulation of signals, and signaling cross-talk. Cytokine Growth Factor Rev. 16, 251-263

92 Sieber, C. et al. (2009) Recent advances in BMP receptor signaling. Cytokine Growth Factor Rev. 20, 343-355

93 Miyazono, K. et al. (2010) Bone morphogenetic protein receptors and signal transduction. J. Biochem. 147, 35-51

94 Ruschke, K. et al. (2012) BMPs are mediators in tissue crosstalk of the regenerating musculoskeletal system. Cell Tissue Res. 347, 521-544

95 Kim, J.H. et al. (2012) BMP9 induces EphrinB2 expression in endothelial cells through an Alk1-BMPRII/ActRII-ID1/ID3-dependent pathway: implications for hereditary hemorrhagic telangiectasia type II. Angiogenesis 15, 497-509

96 Poirier, O. et al. (2012) Inhibition of Apelin expression by Bmp signaling in endothelial cells. Am. J. Physiol. http://dx.doi.org/ 10.1152/ajpcell.00168.2012

97 William, D.A. et al. (2007) Identification of oscillatory genes in somitogenesis from functional genomic analysis of a human mesenchymal stem cell model. Dev. Biol. 305, 172-186

98 Fortini, M.E. (2009) Notch signaling: the core pathway and its posttranslational regulation. Dev. Cell 16, 633-647

99 Pierfelice, T. et al. (2011) Notch in the vertebrate nervous system: an old dog with new tricks. Neuron $69,840-855$ 